\section{Práticas dos trabalhadores de saúde na comunidade nos modelos de atenção básica do Sul e Nordeste do Brasil}

\author{
Health workers' community practices in primary \\ health care models in South and Northeast Brazil
}

\author{
1 Secretaria Municipal de \\ Saúde e Bem Estar de Pelotas, \\ Pelotas, Brasil. \\ 2 Programa de Pós-graduação \\ em Epidemiologia \\ Universidade Federal de \\ Pelotas, Pelotas, Brasil. \\ 3 Centro de Ciências da Vida \\ e da Saúde, Universidade \\ Católica de Pelotas, Pelotas \\ Brasil. \\ 4 Programa de Pós-graduação \\ em Saúde e Comportamento, \\ Universidade Católica de \\ Pelotas, Pelotas, Brasil. \\ 5 Faculdade de Enfermagen \\ e Obstetrícia, Universidade \\ Federal de Pelotas, Pelotas, \\ Brasil. \\ Correspondência \\ A. R. Martins \\ Secretaria Municipal de Saúde \\ e Bem Estar de Pelotas. \\ Rua Dr. Osvaldo Branco de \\ Araújo 111, Pelotas, RS 96085- \\ 355, Brasil. \\ enf.alexandra@hotmail.com
}

\section{Abstract}

In order to verify the prevalence of health workers' participation in health practices in the community, a cross-sectional study was conducted with 3,743 primary care health workers in 41 municipalities with more than 100 thousand inhabitants each in South and Northeast Brazil. Overall prevalence of participation in the community was $62.7 \%$, and was significantly higher in the Northeast and in the Family Health Program (FHP). The most common practice was health education on special holidays, in talks, and in groups. There was a positive association between participation and: female gender, training in the management of chronic diseases, use of protocols, satisfaction with community meetings, and academic health services. Differences in the prevalence of participation by region and health care model reaffirmed the inherent expectations in the historical context of reorganization of primary care. The results indicate that it is necessary to expand participation by health workers in the community, considering that primary care is the level of care that provides the best possibilities for promoting health practices with equity.

Professional Practice; Health Personnel; Primary Health Care

\author{
Alexandra da Rosa Martins 1 \\ Denise Silva da Silveira 1,2 \\ Fernando Vinholes Siqueira 3 \\ Luiz Augusto Facchini 2 \\ Roberto Xavier Piccini 2 \\ Elaine Tomasi 1,4 \\ Elaine Thumé 5 \\ Marilu Correa Soares 5
}

A atenção básica à saúde caracteriza-se por um conjunto de ações no âmbito individual e coletivo, realizadas por equipes multiprofissionais, que visam à promoção e proteção da saúde, à prevenção de agravos, ao diagnóstico, ao tratamento, à reabilitação e à manutenção da saúde 1 . Seus princípios se baseiam no conceito de atenção primária à saúde estabelecidos em Alma Ata 2,3. No Brasil, até o surgimento do Programa Saúde da Família (PSF) em 1994, a atenção básica à saúde organizava-se sobretudo com base em serviços norteados pelos princípios de um modelo que entendia a saúde apenas como a ausência de doença, baseando-se em práticas freqüentemente clientelistas e de conteúdo curativo. A oferta de atenção concentrava-se no indivíduo e suas demandas, desconsiderando a realidade e autonomia locais, o planejamento a partir de perfis epidemiológicos e a participação comunitária 4.

O PSF surgiu para estruturar a atenção básica à saúde nos princípios do Sistema Único de Saúde (SUS). Propõe a reorganização das práticas baseando-se no conhecimento das reais necessidades da comunidade sob sua responsabilidade, para o alcance tanto da integralidade quanto da eqüidade em saúde 5,6,7,8. A estruturação dos serviços com base nas necessidades da população implica a implementação de abordagens mais amplas e complexas do que as centradas no cui- 
dado curativo, que viabilizem a compreensão de como os problemas de saúde se manifestam na população. Nessa perspectiva, a atuação dos trabalhadores de saúde não deve restringir-se apenas à unidade básica de saúde (UBS). É necessário que ocorra também nos domicílios e demais espaços comunitários, permitindo maior contato com as singularidades de cada indivíduo, família e comunidade, de forma a contribuir para as ações efetivas e adaptadas às desigualdades dos grupos sociais e diferentes demandas em saúde 3,9,10,11,12.

Contudo, apenas a implantação do PSF não garante o alcance da mudança do modelo assistencial proposto pelo SUS 9,13,14. Estudos nacionais que avaliaram o grau de utilização das atividades previstas encontraram baixa participação das equipes em grupos e instituições locais com vistas à resolução dos problemas, evidenciando a escassez de atividades participativas com a comunidade. As práticas ainda reiteram a lógica do atendimento clínico, individual e curativo, predominantemente voltadas à assistência $8,13,15,16,17$.

O presente estudo tem o objetivo de verificar a prevalência da realização de práticas de saúde por parte dos trabalhadores na comunidade, assim como identificar as práticas realizadas, em sete estados das regiões Sul e Nordeste do Brasil. Esta pesquisa também verifica alguns fatores associados com o desfecho.

\section{Métodos}

Foi realizado um estudo de delineamento transversal que selecionou uma amostra de 3.743 trabalhadores de saúde de UBS de 41 municípios com mais de 100 mil habitantes dos estados do Rio Grande do Sul e Santa Catarina na Região Sul, Alagoas, Pernambuco, Paraíba, Rio Grande do Norte e Piauí na Região Nordeste do Brasil. Os municípios incluídos na pesquisa compõem os Lotes 2 (Sul e Nordeste) do Estudo de Linha de Base do Projeto de Expansão e Consolidação da Estratégia Saúde da Família (ELB-PROESF), conduzido pela Universidade Federal de Pelotas (UFPel) 17,18,19. Foram identificadas 1.500 UBS, sendo 850 no Nordeste e 650 no Sul. Uma amostra aleatória de 120 UBS foi sorteada em cada uma das regiões, sendo estabelecida uma proporcionalidade com o tamanho da rede básica dos municípios e os modelos de atenção (tradicional e PSF) 20,21,22. No Sul, a amostra foi composta por 69 UBS do PSF e 51 tradicionais, enquanto no Nordeste 79 eram do PSF e 41 tradicionais. Todos os trabalhadores das UBS selecionadas foram convidados a participar do estudo e confrontou-se a amostra obtida com o registro dos profissionais lotados nas UBS.

A coleta de dados ocorreu entre os meses de março a agosto de 2005, com uma equipe de 15 supervisores de campo vinculados ao ELBPROESF/UFPel. Após consentimento, os trabalhadores responderam a um questionário autoaplicado que foi revisado e codificado pela equipe de pesquisa. A digitação dos dados foi feita utilizando-se o programa Epi Info versão 6 (Centers for Disease Control and Prevention, Atlanta, Estados Unidos).

Vários cálculos de tamanho de amostra foram realizados para atender aos objetivos do estudo. Com a amostra obtida (3.743), a margem de erro para a estimativa de prevalência de práticas dos trabalhadores de saúde realizadas na comunidade (estimada em 55\%) foi de 1,6 ponto percentual. Para avaliação de associações, a pesquisa teve poder de $80 \%$ para detectar como significativas razões de prevalência de 1,3 ou maiores, para exposições que afetam de $10-90 \%$ da população estudada, com nível de 95\% de confiança.

Foram consideradas práticas dos trabalhadores de saúde na comunidade as atividades desenvolvidas fora da estrutura física do serviço, não incluindo visitas domiciliares para cuidado clínico de doença ou visita mensal periódica do agente comunitário de saúde (ACS) às famílias, que tenham sido realizadas nos últimos 12 meses.

As variáveis independentes incluídas nas análises para associações foram: (a) sexo; (b) idade em anos (16-29, 30-49 e 50 ou mais); (c) escolaridade (Ensino Fundamental incompleto, Ensino Fundamental completo, Ensino Médio incompleto, Ensino Médio completo, Ensino Superior incompleto e Ensino Superior completo); (d) atividade profissional na UBS (auxiliar e técnico de enfermagem/auxiliar e técnico de consultório dentário, enfermeiro, médico, odontólogo, outro profissional de nível superior e ACS); (e) regime de trabalho precário (não e sim); (f) vinculação a plano de carreira (não e sim); (g) primeiro emprego (não e sim); (h) trabalha em outro emprego (não e sim); (i) tempo de atuação na UBS em tercis (até 24 meses, de 25-108 meses, 109 meses ou mais); (j) capacitação profissional em saúde da mulher (não e sim); (k) capacitação profissional em saúde da criança (não e sim); (l) capacitação profissional no manejo de doenças crônicas (não e sim); (m) utilização de protocolos (não e sim); (n) satisfação com reuniões comunitárias nota de 0 a 10 em tercis $(0-4,0 ; 4,1$ 7,0 e 7,1-10); (o) vinculação da UBS com ensino (não e sim); (p) satisfação do trabalhador com a sua saúde (insatisfeito, indiferente e satisfeito); e (q) transtornos mentais comuns (negativo e 
positivo). A aferição dos transtornos mentais comuns foi realizada usando-se o Self-Report Questionnaire-20 (SRQ-20) 23.

A análise dos dados se deu usando-se o programa Stata 7.0 (Stata Corp., College Station, Estados Unidos). Foram realizadas análises descritivas, bruta e ajustada para toda a amostra, e estratificada de acordo com a região e o modelo de atenção. A existência de diferenças em relação à participação dos trabalhadores em práticas na comunidade foi explorada, na análise bruta, com o uso do teste de Wald para heterogeneidade e tendência linear. A análise ajustada foi realizada por regressão de Poisson com cálculos robustos de razões de prevalências ajustadas, intervalos de 95\% de confiança (IC95\%) e valores de significância usando-se os mesmos testes descritos acima, segundo o pressuposto de que exista uma relação hierárquica entre as variáveis e o desfecho. As variáveis de "a-c" fizeram parte do nível mais distal, as de "d-o" do intermediário e as de "p-q" do proximal. Foram mantidas na análise aquelas variáveis com valor $\mathrm{p} \leq 0,20$ com o objetivo de controle de possíveis fatores de confusão, e foram consideradas significativas aquelas com valor $\mathrm{p} \leq 0,05$.

O Comitê de Ética da Faculdade de Medicina da UFPel aprovou o protocolo do estudo.

\section{Resultados}

Na impossibilidade de saber o número exato de trabalhadores do Sul e do Nordeste, a amostra obtida foi confrontada com o registro dos profissionais lotados nas UBS selecionadas. No Nordeste, a cobertura observada foi de $73 \%$ para os médicos e de $90 \%$ para os demais profissionais. No Sul, a cobertura foi de $93 \%$ para todos os profissionais.

De um total de 3.743 profissionais de saúde incluídos na pesquisa, 1.464 eram do Sul e 2.279 do Nordeste. Desses, 2.433 (65\%) pertenciam às UBS do PSF e 1.310 (35\%) às UBS tradicionais. A prevalência de participação dos trabalhadores de saúde em práticas realizadas na comunidade foi de 62,7\% (IC95\%: 61,1-64,3), sendo significativamente maior no Nordeste (Sul: $57,7 \%$; Nordeste: 65,9\%; $\mathrm{p}<0,001$ ) e no modelo PSF (tradicional: $37,7 \%$; PSF: 75,5\%; p < 0,001). A taxa de não resposta foi de $5,4 \%$, sendo de $6,2 \%$ no Sul, $4,9 \%$ no Nordeste, $3,5 \%$ no PSF e 8,9\% no tradicional.

Entre as práticas desenvolvidas na comunidade no último ano, $67 \%$ dos trabalhadores destacaram realizar atividades de educação em saúde aproveitando as datas festivas, os encontros para palestras e também as reuniões em grupos; $17 \%$ o envolvimento em atividades não especificadas com controle social e ação social; e $16 \%$ mencionaram atividades e procedimentos específicos, tais como aplicação de vacinas e pesagem de crianças durante campanhas, escovação e aplicação de flúor, e cadastramento do Programa Bolsa Família. Em ambas as regiões e modelos de atenção também houve predomínio da participação dos trabalhadores em práticas de educação em saúde nas datas festivas, nas palestras e nos grupos. A segunda prática mais referida foi atividade não especificada junto ao controle social e às ações sociais no Sul (20,5\%) e no PSF (16,8\%), e atividades e procedimentos específicos no Nordeste $(16,9 \%)$ e no modelo tradicional $(29,4 \%)$ (Figura 1).

Quanto à atividade profissional, os odontólogos foram os trabalhadores que mais se envolveram nas práticas de educação em saúde em datas festivas, palestras ou grupos $(77,3 \%)$, seguidos dos ACS (68,9\%), auxiliares ou técnicos de enfermagem e consultório dentário $(64,2 \%)$, médicos e enfermeiros (61,9\% e 61,6\%, respectivamente) e outros profissionais de nível superior (57,9\%).

A Tabela 1 descreve a amostra de profissionais de acordo com as variáveis independentes incluídas no estudo. Em ambas as regiões e modelos de atenção houve predomínio de trabalhadores do sexo feminino. As mulheres estavam em proporção significativamente maior no Nordeste $(84,3 \%)$ e no PSF (85\%). A média de idade foi de 37,3 anos ( $\mathrm{DP}=10,0$ ), distribuindo-se de forma semelhante ente as regiões (Sul: 37,2 anos, DP = 10,2; Nordeste: 37,4 anos, DP $=9,8$ ), com maior concentração de trabalhadores na categoria de 30-49 anos $(61,2 \%)$, tanto no Sul quanto no Nordeste. O predomínio dessa faixa etária se mantém nos modelos de atenção, destacando-se a maior freqüência nas UBS tradicionais $(62,9 \%$; $\mathrm{p}<0,001)$. A escolaridade preponderante foi ensino médio completo $(43,1 \%)$, sendo significativamente maior no Nordeste $(47,8 \%)$ e no PSF $(45,7 \%)$.

Na distribuição da amostra por atividade profissional na UBS observou-se maior prevalência de ACS (41\%), seguidos por auxiliares/técnicos de enfermagem e consultório dentário $(25,1 \%)$ e médicos (13,9\%). De acordo com as regiões encontraram-se proporções significativamente maiores de odontólogos, outros profissionais de nível superior e agentes comunitários de saúde no Nordeste, e de auxiliares/técnicos de enfermagem e consultório dentário, médicos e enfermeiros no Sul. No modelo tradicional houve uma maior proporção de auxiliares/técnicos de enfermagem e consultório dentário, médicos, odontólogos e outros profissionais de nível superior. Já no PSF observou-se uma maior proporção de ACS e enfermeiros. 
Figura 1

Prevalência dos tipos de práticas realizadas pelos trabalhadores de saúde da atenção básica na comunidade total, de acordo com a região e o modelo de atenção. Brasil, 2005.

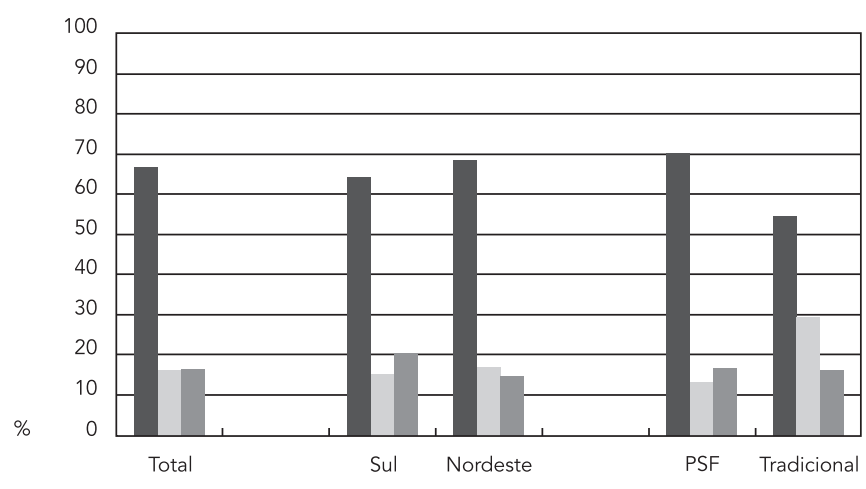

Educação em saúde: datas festivas/grupos

Práticas junto ao controle social/às ações sociais $\square$ Práticas específicas/procedimentos

PSF: Programa Saúde da Família

Tabela 1

Descrição das variáveis independentes na amostra de trabalhadores de unidades básicas de saúde (UBS) total e estratificada por região e modelo de atenção. Brasil, 2005

\begin{tabular}{|c|c|c|c|c|c|c|c|c|c|c|c|c|}
\hline \multirow[t]{2}{*}{ Variáveis } & \multicolumn{2}{|c|}{ Total } & \multicolumn{2}{|c|}{ Sul } & \multicolumn{2}{|c|}{ Nordeste } & \multirow{2}{*}{$\begin{array}{l}\text { Valor } \\
\text { de p }\end{array}$} & \multicolumn{2}{|c|}{ PSF } & \multicolumn{2}{|c|}{ Tradicional } & \multirow{2}{*}{$\begin{array}{l}\text { Valor } \\
\text { de } p\end{array}$} \\
\hline & $\%$ & $n$ & $\%$ & $n$ & $\%$ & $\mathbf{n}$ & & $\%$ & $\mathrm{n}$ & $\%$ & $\mathrm{n}$ & \\
\hline Sexo & & & & & & & 0,04 & & & & & $<0,001$ \\
\hline Masculino & 16,7 & 620 & 18,3 & 267 & 15,7 & 353 & & 15,0 & 362 & 20,0 & 258 & \\
\hline Feminino & 83,3 & 3.087 & 81,7 & 1.192 & 84,3 & 1.895 & & 85,0 & 2.053 & 80,0 & 1.034 & \\
\hline Idade (anos) & & & & & & & $0,31 *$ & & & & & $<0,001$ * \\
\hline $16-29$ & 25,8 & 935 & 27,1 & 394 & 25,0 & 559 & & 29,7 & 713 & 18,7 & 240 & \\
\hline $30-49$ & 61,2 & 2.257 & 59,8 & 868 & 62,1 & 1.389 & & 60,4 & 1.452 & 62,9 & 805 & \\
\hline 50 ou mais & 13,0 & 479 & 13,1 & 190 & 12,9 & 289 & & 9,9 & 283 & 18,7 & 241 & \\
\hline Escolaridade & & & & & & & $<0,001$ * & & & & & $<0,001$ * \\
\hline Ensino Fundamental incompleto & 1,8 & 66 & 2,2 & 32 & 1,5 & 34 & & 1,9 & 46 & 1,5 & 20 & \\
\hline Ensino Fundamental completo & 4,4 & 162 & 4,4 & 64 & 4,3 & 98 & & 5,1 & 124 & 2,9 & 38 & \\
\hline Ensino Médio incompleto & 7,4 & 275 & 9,2 & 134 & 6,2 & 141 & & 8,6 & 209 & 5,1 & 66 & \\
\hline Ensino Médio completo & 43,1 & 1.604 & 35,9 & 523 & 47,8 & 1.081 & & 45,7 & 1.104 & 38,5 & 500 & \\
\hline Ensino Superior incompleto & 6,3 & 236 & 7,4 & 107 & 5,7 & 129 & & 6,5 & 158 & 6,0 & 78 & \\
\hline Ensino Superior completo & 37,0 & 1.375 & 40,9 & 595 & 34,4 & 780 & & 32,1 & 777 & 46,0 & 598 & \\
\hline Atividade profissional na UBS & & & & & & & $<0,001$ & & & & & $<0,001$ \\
\hline Agentes comunitários de saúde & 41,0 & 1.536 & 32,7 & 478 & 46,3 & 1.058 & & 48,6 & 1.182 & 27,0 & 354 & \\
\hline Auxiliar/Técnico de enfermagem e & 25,1 & 938 & 29,2 & 428 & 22,4 & 510 & & 22,6 & 550 & 29,6 & 388 & \\
\hline \multicolumn{13}{|l|}{$\begin{array}{l}\text { Auxiliar/Técnico de consultório } \\
\text { dentário }\end{array}$} \\
\hline Médico & 13,9 & 521 & 18,2 & 266 & 11,2 & 255 & & 10,7 & 260 & 19,9 & 261 & \\
\hline Enfermeiro & 9,1 & 341 & 11,1 & 162 & 7,9 & 255 & & 10,0 & 243 & 7,5 & 98 & \\
\hline Odontólogo & 6,4 & 239 & 5,9 & 87 & 6,7 & 179 & & 5,8 & 141 & 7,5 & 98 & \\
\hline Outros de nível superior & 4,5 & 168 & 2,9 & 43 & 5,5 & 152 & & 2,3 & 57 & 8,5 & 111 & \\
\hline
\end{tabular}

(continua) 
Tabela 1 (continuação)

\begin{tabular}{|c|c|c|c|c|c|c|c|c|c|c|c|c|}
\hline \multirow[t]{2}{*}{ Variáveis } & \multicolumn{2}{|c|}{ Total } & \multicolumn{2}{|c|}{ Sul } & \multicolumn{2}{|c|}{ Nordeste } & \multirow{2}{*}{$\begin{array}{l}\text { Valor } \\
\text { de } p\end{array}$} & \multicolumn{2}{|c|}{ PSF } & \multicolumn{2}{|c|}{ Tradicional } & \multirow{2}{*}{$\begin{array}{l}\text { Valor } \\
\text { de } p\end{array}$} \\
\hline & $\%$ & $\mathrm{n}$ & $\%$ & $\mathbf{n}$ & $\%$ & $\mathbf{n}$ & & $\%$ & $\mathbf{n}$ & $\%$ & $\mathbf{n}$ & \\
\hline Regime de trabalho precarizado & & & & & & & $<0,001$ & & & & & $<0,001$ \\
\hline Não & 61,2 & 2.210 & 73,1 & 1.034 & 53,6 & 1.176 & & 58,0 & 1.362 & 67,2 & 848 & \\
\hline $\operatorname{Sim}$ & 38,8 & 1.399 & 26,9 & 380 & 46,4 & 1.019 & & 42,0 & 985 & 32,8 & 414 & \\
\hline Vinculação ao plano de carreira & & & & & & & 0,15 & & & & & $<0,001$ \\
\hline Não & 85,1 & 2.900 & 86,3 & 1.123 & 84,5 & 1.777 & & 89,4 & 2.022 & 76,7 & 878 & \\
\hline Sim & 14,9 & 506 & 13,7 & 179 & 15,5 & 327 & & 10,6 & 239 & 23,3 & 267 & \\
\hline Primeiro emprego & & & & & & & $<0,001$ & & & & & 0,75 \\
\hline Não & 75,6 & 2.800 & 83,4 & 1.204 & 70,7 & 1.596 & & 75,8 & 1.826 & 75,3 & 974 & \\
\hline Sim & 24,4 & 902 & 16,6 & 240 & 29,3 & 662 & & 24,2 & 583 & 24,7 & 319 & \\
\hline Outro emprego além do atual na UBS & & & & & & & 0,08 & & & & & $<0,001$ \\
\hline Não & 76,1 & 2.805 & 77,7 & 1.120 & 75,2 & 1.685 & & 81,9 & 1.974 & 65,2 & 831 & \\
\hline Sim & 23,9 & 879 & 22,3 & 322 & 24,8 & 557 & & 18,1 & 435 & 34,8 & 444 & \\
\hline Tempo de atuação na UBS em tercis & & & & & & & $<0,001$ * & & & & & $<0,001$ * \\
\hline Até 24 meses & 52,0 & 1.881 & 62,7 & 892 & 45,1 & 989 & & 57,5 & 1.368 & 41,6 & 513 & \\
\hline 25-108 meses & 37,2 & 1.344 & 29,3 & 417 & 42,3 & 927 & & 36,6 & 870 & 38,4 & 474 & \\
\hline 109 meses ou mais & 10,8 & 389 & 7,9 & 113 & 12,6 & 276 & & 6,0 & 142 & 20,0 & 247 & \\
\hline $\begin{array}{l}\text { Capacitação profissional em saúde da } \\
\text { mulher }\end{array}$ & & & & & & & $<0,001$ & & & & & $<0,001$ \\
\hline Não & 44,9 & 1.192 & 40,0 & 422 & 48,2 & 770 & & 41,8 & 746 & 51,4 & 446 & \\
\hline $\operatorname{Sim}$ & 55,1 & 1.460 & 60,0 & 633 & 51,8 & 827 & & 58,2 & 1.039 & 48,6 & 421 & \\
\hline $\begin{array}{l}\text { Capacitação profissional em saúde da } \\
\text { criança }\end{array}$ & & & & & & & 0,02 & & & & & $<0,001$ \\
\hline Não & 42,3 & 1.156 & 40,8 & 432 & 43,3 & 724 & & 38,1 & 705 & 51,0 & 451 & \\
\hline $\operatorname{Sim}$ & 57,7 & 1.576 & 59,2 & 628 & 56,7 & 948 & & 61,9 & 1.143 & 49,0 & 433 & \\
\hline $\begin{array}{l}\text { Capacitação profissional no manejo de } \\
\text { doenças crônicas }\end{array}$ & & & & & & & 0,05 & & & & & $<0,001$ \\
\hline Não & 42,0 & 1.145 & 39,7 & 425 & 43,5 & 720 & & 37,9 & 698 & 50,5 & 447 & \\
\hline $\operatorname{Sim}$ & 58,0 & 1.583 & 60,3 & 646 & 56,5 & 937 & & 62,1 & 1.144 & 49,5 & 439 & \\
\hline Utilização de protocolos pelo profissional & & & & & & & $<0,001$ & & & & & $<0,001$ \\
\hline Não & 52,6 & 1.859 & 60,9 & 839 & 47,2 & 1.020 & & 50,1 & 1.165 & 57,2 & 694 & \\
\hline Sim & 47,4 & 1.678 & 39,1 & 538 & 52,8 & 1.140 & & 49,9 & 1.159 & 42,8 & 519 & \\
\hline Satisfação com as reuniões comunitárias & & & & & & & 0,11 * & & & & & $<0,001$ * \\
\hline notas $0-10$ em tercis & & & & & & & & & & & & \\
\hline $0-4,0$ & 33,7 & 1.116 & 33,5 & 436 & 33,9 & 680 & & 27,1 & 630 & 49,4 & 486 & \\
\hline $4,1-7,0$ & 37,7 & 1.248 & 36,0 & 469 & 38,8 & 779 & & 40,4 & 939 & 31,4 & 309 & \\
\hline $7,1-10,0$ & 28,6 & 946 & 30,5 & 397 & 27,3 & 549 & & 32,6 & 758 & 19,1 & 188 & \\
\hline Vinculação da UBS com ensino & & & & & & & 0,02 & & & & & $<0,001$ \\
\hline Não & 48,0 & 1.743 & 45,6 & 621 & 49,5 & 1.122 & & 40,0 & 944 & 63,1 & 799 & \\
\hline Sim & 52,0 & 1.886 & 54,4 & 742 & 50,5 & 1.144 & & 60,0 & 1.418 & 36,9 & 468 & \\
\hline Satisfação com a saúde & & & & & & & $<0,001 *$ & & & & & 0,06 * \\
\hline Insatisfeito & 10,0 & 371 & 12,0 & 173 & 8,8 & 198 & & 9,9 & 238 & 10,4 & 133 & \\
\hline Indiferente & 23,1 & 854 & 24,2 & 348 & 22,4 & 506 & & 24,3 & 586 & 20,9 & 268 & \\
\hline Satisfeito & 66,9 & 2.469 & 63,7 & 916 & 68,8 & 1.553 & & 65,8 & 1.585 & 68,8 & 884 & \\
\hline Transtornos mentais comuns (SRQ-20) & & & & & & & 0,41 & & & & & 0,06 \\
\hline Negativo & 84,5 & 2.833 & 85,2 & 1.121 & 84,1 & 1.712 & & 83,7 & 1.826 & 86,2 & 1.010 & \\
\hline Positivo & 15,5 & 518 & 14,8 & 195 & 15,9 & 323 & & 16,3 & 356 & 13,8 & 162 & \\
\hline
\end{tabular}

PSF: Programa Saúde da Família; SRQ-20: Self-Report Questionnaire-20.

* Teste de Wald para tendência. 
O regime de trabalho precário foi uma realidade para $38,8 \%$ dos trabalhadores, e foi significativamente maior entre os do Nordeste $(46,4 \%)$. Constatou-se que o PSF apresenta uma maior prevalência de trabalho precário (42\%). Da amostra estudada apenas $14,9 \%$ possuíam vinculação ao plano de carreira, ocorrendo uma diferença significativa da prevalência na comparação do modelo tradicional $(23,3 \%)$ em relação ao PSF $(10,6 \%)-p<0,001$. A atuação na UBS como primeiro emprego foi referida por $24,4 \%$ dos trabalhadores, $16,6 \%$ no Sul e $29,3 \%$ no Nordeste ( $\mathrm{p}<$ $0,001)$, sem haver diferença significativa entre os modelos de atenção. A maioria dos trabalhadores $(76,1 \%)$ não possuía outro emprego, especialmente aqueles vinculados às UBS do PSF, e mais da metade tinha uma atuação em UBS por até 24 meses. Comparativamente ao Nordeste, no Sul a prevalência do tempo de atuação por até 24 meses foi significativamente maior. O mesmo ocorreu na comparação do tempo de atuação entre os modelos de atenção (PSF: 57,5\%; tradicional: $41,6 \%$; $<<0,001)$.

Pelo menos a metade dos trabalhadores estudados recebeu capacitação em saúde da mulher $(55,1 \%)$, saúde da criança $(57,7 \%)$ e manejo de doenças crônicas (58\%). Comparando-se os dois lotes, observou-se que os trabalhadores do Sul realizaram mais capacitações do que os do Nordeste. Em geral, a prevalência de trabalhadores capacitados foi significativamente maior no PSF, sendo de $62,1 \%$ para o manejo de doenças crônicas, de $61,9 \%$ na área de saúde da criança e de $58,2 \%$ na de saúde da mulher. Quanto aos protocolos, apenas $47,4 \%$ de todos os trabalhadores fizeram uso deles para a realização de suas atividades, destacando-se a maior utilização pelos profissionais da Região Nordeste (52,8\%; $\mathrm{p}<0,001)$ e entre os vinculados às UBS do PSF $(49,9 \% ; \mathrm{p}<0,001)$.

Na avaliação da satisfação dos trabalhadores com reuniões comunitárias, a nota média atribuída foi 5,3 (DP = 3,0). Mais de um terço $(37,7 \%)$ da amostra concentrou-se na categoria que avaliou essa satisfação com notas entre 4,1-7,0, sendo a distribuição semelhante entre as regiões e maior no PSF (PSF: 40,4\%; tradicional: $31,4 \%$; $\mathrm{p}<0,001$ ). Com relação à autopercepção sobre a saúde, $66,9 \%$ dos trabalhadores referiram-se satisfeitos, com maior prevalência no Nordeste $(68,8 \%)$ em comparação ao Sul $(63,7 \%)-p=0,001$, sem haver diferença significativa entre os modelos de atenção. A prevalência de transtornos mentais comuns avaliada utilizando-se o SRQ-20 foi de $15,5 \%$ e distribuiu-se de forma semelhante entre os estratos (região e modelo).

A Tabela 2 apresenta a associação bruta e ajustada entre as práticas realizadas na comu- nidade e as variáveis independentes para toda a amostra estudada. Pôde-se verificar após a análise ajustada que as práticas na comunidade foram mais freqüentes entre os trabalhadores do sexo feminino (RP = 1,17; IC95\%: 1,01-1,34), capacitados para o manejo de doenças crônicas (RP = 1,30; IC95\%: 1,20-1,42), que utilizavam protocolos para exercer as atividades na UBS $(\mathrm{RP}=1,20$; IC95\%: 1,14-1,27), mais satisfeitos com reuniões comunitárias (RP = 1,20; IC95\%: 1,12-1,29) e que trabalhavam em UBS vinculadas ao ensino ( $\mathrm{RP}=$ 1,12; IC95\%: 1,05-1,18). Por outro lado, essas práticas foram menos freqüentes entre os trabalhadores com 50 anos ou mais de idade, de escolaridade superior completa, vinculados aos planos de carreira e que possuíam outro emprego além do atual na UBS.

A Tabela 3 apresenta a análise estratificada para as regiões Sul e Nordeste. No Sul, as práticas dos trabalhadores de saúde na comunidade foram significativamente mais prevalentes entre trabalhadores do sexo feminino $(\mathrm{RP}=1,17$; IC95\%: 1,01-1,34), odontólogos ( $R P=1,45$; IC95\%: 1,131,87), capacitados na atenção à saúde da criança (RP = 1,20; IC95\%: 1,01-1,42) e no manejo de doenças crônicas (RP = 1,33; IC95\%: 1,11-1,59), e mais satisfeitos com reuniões comunitárias $(\mathrm{RP}=$ 1,57; IC95\%: 1,35-1,82). Inversamente, a probabilidade de realização de práticas na comunidade foi menor entre trabalhadores com 50 anos ou mais de idade, de escolaridade média completa, superior incompleta e completa, vinculados ao plano de carreira e com outro emprego além do atual na UBS. Já no Nordeste, associaram-se positivamente ao desfecho as variáveis: capacitação no manejo de doenças crônicas $(\mathrm{RP}=1,29$; IC95\%: 1,19-1,39), utilização de protocolos (RP = 1,20; IC95\%: 1,12-1,28) e UBS vinculada ao ensino (RP = 1,21; IC95\%: 1,13-1,29). De forma contrária, as práticas na comunidade no Nordeste foram menos freqüentes ente os trabalhadores mais escolarizados, de 30 anos ou mais de idade, vinculados ao plano de carreira e que possuíam outro emprego além do atual na UBS.

$\mathrm{Na}$ análise bruta e ajustada da amostra de trabalhadores conforme o modelo de atenção encontrou-se que, no PSF, a prevalência de práticas na comunidade foi mais freqüente entre os odontólogos (RP = 1,28; IC95\%: 1,15-1,43), aqueles com tempo de atuação na UBS maior que dois anos (RP = 1,08; IC95\%: 1,02-1,14) e menor que 10 anos $(\mathrm{RP}=1,15$; IC95\%: 1,04$1,28)$, os capacitados no manejo de doenças crônicas (RP = 1,19; IC95\%: 1,12-1,27), que utilizavam protocolos para realizar suas atividades na UBS (RP = 1,14; IC95\%: 1,08-1,20), os mais satisfeitos com a reunião comunitária (RP = 1,11; IC95\%: 1,04-1,19) e os satisfeitos com a 
Tabela 2

Prevalência de atividades na comunidade conforme as variáveis independentes, análise bruta e ajustada da amostra de trabalhadores de unidades básicas de saúde (UBS) de municípios das regiões Sul e Nordeste. Brasil, 2005.

\begin{tabular}{|c|c|c|c|c|c|}
\hline \multirow[t]{2}{*}{ Níveis/Variáveis } & \multicolumn{3}{|c|}{ Análise bruta } & \multicolumn{2}{|c|}{ Análise ajustada } \\
\hline & $\%$ & RP (IC95\%) & Valor de $p$ & RP (IC95\%) & Valor de $\mathrm{p}$ \\
\hline \multicolumn{6}{|l|}{ Nível 1} \\
\hline Sexo & & & $<0,001$ & & 0,02 \\
\hline Masculino & 55,2 & 1,00 & & 1,00 & \\
\hline Feminino & 64,4 & $1,17(1,08-1,26)$ & & $1,17(1,01-1,34)$ & \\
\hline Idade (anos) & & & $<0,001$ * & & $<0,001$ * \\
\hline $16-29$ & 68,5 & 1,00 & & 1,00 & \\
\hline $30-49$ & 63,1 & $0,92(0,87-0,97)$ & & $0,94(0,85-1,04)$ & \\
\hline 50 ou mais & 49,3 & $0,72(0,65-0,80)$ & & $0,76(0,63-0,90)$ & \\
\hline Escolaridade & & & $<0,001 *$ & & $<0,001$ * \\
\hline Ensino Fundamental incompleto & 69,5 & 1,00 & & 1,00 & \\
\hline Ensino Fundamental completo & 74,2 & $1,07(0,88-1,30)$ & & $1,02(0,85-1,24)$ & \\
\hline Ensino Médio incompleto & 71,3 & $1,02(0,85-1,24)$ & & $0,98(0,82-1,17)$ & \\
\hline Ensino Médio completo & 67,5 & $0,97(0,82-1,15)$ & & $0,92(0,78-1,08)$ & \\
\hline Ensino Superior incompleto & 63,2 & $0,91(0,75-1,11)$ & & $0,84(0,69-1,01)$ & \\
\hline Ensino Superior completo & 54,0 & $0,78(0,65-0,93)$ & & $0,76(0,64-0,90)$ & \\
\hline \multicolumn{6}{|l|}{ Nível 2} \\
\hline Atividade profissional na UBS & & & $<0,001$ & & 0,70 \\
\hline Agentes comunitários de saúde & 79,4 & 1,00 & & 1,00 & \\
\hline Auxiliar/Técnico de enfermagem e & 47,2 & $0,60(0,55-0,64)$ & & $0,77(0,69-0,87)$ & \\
\hline \multicolumn{6}{|l|}{ Auxiliar/Técnico de consultório dentário } \\
\hline Médico & 40,0 & $0,50(0,45-0,56)$ & & $0,74(0,63-0,87)$ & \\
\hline Enfermeiro & 68,0 & $0,86(0,79-0,93)$ & & $0,97(0,87-1,09)$ & \\
\hline Odontólogo & 64,0 & $0,81(0,73-0,89)$ & & $1,17(1,01-1,34)$ & \\
\hline Outros de nível superior & 45,3 & $0,57(0,48-0,68)$ & & $0,73(0,53-0,97)$ & \\
\hline Regime de trabalho precarizado & & & $<0,001$ & & 0,42 \\
\hline Não & 56,4 & 1,00 & & 1,00 & \\
\hline Sim & 72,1 & $1,28(1,22-1,35)$ & & $1,02(0,97-1,08)$ & \\
\hline Vinculação ao plano de carreira & & & $<0,001$ & & $<0,001$ \\
\hline Não & 66,7 & 1,00 & & 1,00 & \\
\hline Sim & 48,0 & $0,72(0,65-0,79)$ & & $0,81(0,72-0,90)$ & \\
\hline Primeiro emprego & & & 0,74 & & 0,69 \\
\hline Não & 62,8 & 1,00 & & 1,00 & \\
\hline Sim & 62,2 & $0,99(0,93-1,05)$ & & $0,99(0,92-1,06)$ & \\
\hline Outro emprego além do atual na UBS & & & $<0,001$ & & $<0,001$ \\
\hline Não & 68,9 & 1,00 & & 1,00 & \\
\hline $\operatorname{Sim}$ & 43,1 & $0,63(0,58-0,68)$ & & $0,82(0,74-0,90)$ & \\
\hline Tempo de atuação na UBS em tercis & & & 0,001 * & & 0,71 * \\
\hline Até 24 meses & 63,7 & 1,00 & & 1,00 & \\
\hline 25-108 meses & 66,4 & $1,04(0,99-1,10)$ & & $1,04(0,98-1,10)$ & \\
\hline 109 meses ou mais & 48,0 & $0,75(0,67-0,84)$ & & $0,98(0,86-1,11)$ & \\
\hline Capacitação profissional em saúde da & & & $<0,001$ & & 0,94 \\
\hline \multicolumn{6}{|l|}{ mulher } \\
\hline Não & 52,5 & 1,00 & & 1,00 & \\
\hline $\operatorname{Sim}$ & 74,7 & $1,42(1,34-1,52)$ & & $1,00(0,90-1,11)$ & \\
\hline
\end{tabular}

(continua) 
Tabela 2 (continuação)

\begin{tabular}{|c|c|c|c|c|c|}
\hline \multirow[t]{2}{*}{ Níveis/Variáveis } & \multicolumn{3}{|c|}{ Análise bruta } & \multicolumn{2}{|c|}{ Análise ajustada } \\
\hline & $\%$ & RP (IC95\%) & Valor de $p$ & RP (IC95\%) & Valor de $p$ \\
\hline Capacitação profissional em saúde & & & $<0,001$ & & 0,12 \\
\hline \multicolumn{6}{|l|}{ da criança } \\
\hline Não & 51,4 & 1,00 & & 1,00 & \\
\hline Sim & 75,7 & $1,47(1,38-1,57)$ & & $1,07(0,98-1,16)$ & \\
\hline Capacitação profissional no manejo & & & $<0,001$ & & $<0,001$ \\
\hline \multicolumn{6}{|l|}{ de doenças crônicas } \\
\hline Não & 49,2 & 1,00 & & 1,00 & \\
\hline Sim & 75,5 & $1,53(1,43-1,64)$ & & $1,30(1,20-1,42)$ & \\
\hline Utilização de protocolos pelo profissional & & & $<0,001$ & & $<0,001$ \\
\hline Não & 56,8 & 1,00 & & 1,00 & \\
\hline $\operatorname{Sim}$ & 69,2 & $1,22(1,16-1,28)$ & & $1,20(1,14-1,27)$ & \\
\hline Satisfação com as reuniões comunitárias & & & $<0,001$ * & & $<0,001$ * \\
\hline \multicolumn{6}{|l|}{ notas $0-10$ em tercis } \\
\hline $0-4,0$ & 54,8 & 1,00 & & 1,00 & \\
\hline $4,1-7,0$ & 70,2 & $1,28(1,20-1,37)$ & & $1,16(1,07-1,24)$ & \\
\hline $7,1-10,0$ & 76,3 & $1,39(1,30-1,49)$ & & $1,20(1,12-1,29)$ & \\
\hline Vinculação da UBS com ensino & & & 0,03 & & $<0,001$ \\
\hline Não & 60,8 & 1,00 & & 1,00 & \\
\hline Sim & 64,4 & $1,06(1,01-1,12)$ & & $1,12(1,05-1,18)$ & \\
\hline \multicolumn{6}{|l|}{ Nível 3} \\
\hline Satisfação com a saúde & & & 0,10 * & & 0,16 * \\
\hline Insatisfeito & 57,5 & 1,00 & & 1,00 & \\
\hline Indiferente & 63,2 & $1,10(0,99-1,22)$ & & $1,04(0,93-1,16)$ & \\
\hline Satisfeito & 63,4 & $1,10(1,00-1,21)$ & & $1,07(0,96-1,18)$ & \\
\hline Transtornos mentais comuns (SRQ-20) & & & 0,51 & & 0,41 \\
\hline Negativo & 62,2 & 1,00 & & 1,00 & \\
\hline Positivo & 63,8 & $1,02(0,95-1,10)$ & & $1,03(0,95-1,13)$ & \\
\hline
\end{tabular}

IC95\%: intervalo de 95\% de confiança; RP: razão de prevalência; SRQ-20: Self-Report Questionnaire-20.

* Teste de Wald para tendência.

sua saúde (RP = 1,15; IC95\%: 1,04-1,29). Nesse sentido, no modelo tradicional, o destaque foi para os trabalhadores capacitados no manejo de doenças crônicas $(\mathrm{RP}=1,66$; IC95\%: $1,23-2,23)$, que utilizavam protocolos ( $\mathrm{RP}=$ 1,31; IC95\%: 1,11-1,56) e trabalhavam em UBS de ensino ( $\mathrm{RP}=1,32$; IC95\%: 1,12-1,56). As práticas na comunidade foram menos freqüentes entre os auxiliares e técnicos de enfermagem e consultório dentário do PSF, e entre os trabalhadores mais escolarizados, com outro emprego além do atual na UBS e de 30 ou mais anos de idade, das UBS tradicionais (Tabela 4).

\section{Discussão}

A proposta de olhar para a saúde da população assegurando-se tanto a integralidade quanto a eqüidade, exige que a atuação dos trabalhadores da atenção básica à saúde não se restrinja apenas às atividades realizadas na UBS. Esses novos formatos de produção de ações de saúde indicam que as práticas profissionais devem ocorrer também nos domicílios e demais espaços comunitários. A partir do contato com as singularidades de cada indivíduo, família e comunidade é possível não apenas organizar ações mais flexíveis e adaptadas às particularidades dos grupos sociais como também enfrentar a incorporação das heterogeneidades, das desigualdades sociais e diferentes necessidades de saúde 6,8,11,14,24.

A prevalência de participação dos trabalhadores em práticas de saúde na comunidade encontrada neste estudo foi de $62,7 \%$. Embora pareça elevada, há de se considerar que foi investigada por um período de 12 meses e com diferenciais acentuados entre a atenção básica 
Tabela 3

Prevalência de atividades na comunidade, análise bruta e ajustada da amostra de trabalhadores das unidades básicas de saúde (UBS) conforme as regiões Sul e Nordeste. Brasil, 2005.

\begin{tabular}{|c|c|c|c|c|c|c|c|c|c|c|}
\hline \multirow[t]{3}{*}{ Níveis/Variáveis } & \multicolumn{5}{|c|}{ Região Sul } & \multicolumn{5}{|c|}{ Região Nordeste } \\
\hline & \multicolumn{3}{|c|}{ Análise bruta } & \multicolumn{2}{|c|}{ Análise ajustada } & \multicolumn{3}{|c|}{ Análise bruta } & \multicolumn{2}{|c|}{ Análise ajustada } \\
\hline & $\%$ & $\begin{array}{c}\mathrm{RP} \\
\text { (IC95\%) }\end{array}$ & $\begin{array}{l}\text { Valor } \\
\text { de } p\end{array}$ & $\begin{array}{c}\mathrm{RP} \\
\text { (IC95\%) }\end{array}$ & $\begin{array}{l}\text { Valor } \\
\text { de } p\end{array}$ & $\%$ & $\begin{array}{c}\text { RP } \\
\text { (IC95\%) }\end{array}$ & $\begin{array}{l}\text { Valor } \\
\text { de } p\end{array}$ & $\begin{array}{c}\text { RP } \\
\text { (IC95\%) }\end{array}$ & $\begin{array}{l}\text { Valor } \\
\text { de } p\end{array}$ \\
\hline \multicolumn{11}{|l|}{ Nível 1} \\
\hline Sexo & & & $<0,001$ & & 0,04 & & & 0,07 & & 0,22 \\
\hline Masculino & 46,3 & 1,00 & & 1,00 & & 61,6 & 1,00 & & 1,00 & \\
\hline Feminino & 60,2 & $\begin{array}{c}1,30 \\
(1,12-1,50)\end{array}$ & & $\begin{array}{c}1,17 \\
(1,01-1,34)\end{array}$ & & 67,0 & $\begin{array}{c}1,09 \\
(0,99-1,19)\end{array}$ & & $\begin{array}{c}1,06 \\
(0,97-1,16)\end{array}$ & \\
\hline Idade (anos) & & & $<0,001$ * & & 0,003 * & & & $<0,001$ * & & $<0,001$ * \\
\hline $16-29$ & 62,3 & 1,00 & & 1,00 & & 72,8 & 1,00 & & 1,00 & \\
\hline $30-49$ & 58,7 & $\begin{array}{c}0,93 \\
(0,85-1,03)\end{array}$ & & $\begin{array}{c}0,94 \\
(0,85-1,04)\end{array}$ & & 66,2 & $\begin{array}{c}0,91 \\
(0,85-0,97)\end{array}$ & & $\begin{array}{c}0,91 \\
(0,86-0,97)\end{array}$ & \\
\hline 50 ou mais & 45,7 & $\begin{array}{c}0,73 \\
(0,61-0,88)\end{array}$ & & $\begin{array}{c}0,76 \\
(0,63-0,90)\end{array}$ & & 51,7 & $\begin{array}{c}0,71 \\
(0,63-0,81)\end{array}$ & & $\begin{array}{c}0,73 \\
(0,65-0,84)\end{array}$ & \\
\hline Escolaridade & & & $<0,001$ * & & $<0,001$ * & & & $<0,001$ * & & $<0,001$ * \\
\hline Ensino Fundamental incompleto & 75,7 & 1,00 & & 1,00 & & 63,3 & 1,00 & & 1,00 & \\
\hline Ensino Fundamental completo & 72,9 & $\begin{array}{c}0,96 \\
(0,74-1,24)\end{array}$ & & $\begin{array}{c}0,95 \\
(0,73-1,22)\end{array}$ & & 75,0 & $\begin{array}{c}1,18 \\
(0,88-1,59)\end{array}$ & & $\begin{array}{c}1,15 \\
(0,86-1,55)\end{array}$ & \\
\hline Ensino Médio incompleto & 65,9 & $\begin{array}{c}0,87 \\
(0,68-1,11)\end{array}$ & & $\begin{array}{c}0,85 \\
(0,67-1,07)\end{array}$ & & 76,3 & $\begin{array}{c}1,20 \\
(0,90-1,61)\end{array}$ & & $\begin{array}{c}1,15 \\
(0,86-1,53)\end{array}$ & \\
\hline Ensino Médio completo & 60,5 & $\begin{array}{c}0,80 \\
0,64-0,99)\end{array}$ & & $\begin{array}{c}0,77 \\
(0,63-0,96)\end{array}$ & & 70,8 & $\begin{array}{c}1,12 \\
(0,85-1,47)\end{array}$ & & $\begin{array}{c}1,06 \\
(0,81-1,39)\end{array}$ & \\
\hline Ensino Superior incompleto & 58,2 & $\begin{array}{c}0,77 \\
(0,59-1,00)\end{array}$ & & $\begin{array}{c}0,72 \\
(0,55-0,94)\end{array}$ & & 67,2 & $\begin{array}{c}1,06 \\
(0,79-1,43)\end{array}$ & & $\begin{array}{c}0,98 \\
(0,73-1,32)\end{array}$ & \\
\hline Ensino Superior completo & 51,1 & $\begin{array}{c}0,67 \\
(0,54-0,84)\end{array}$ & & $\begin{array}{c}0,69 \\
(0,55-0,56)\end{array}$ & & 56,3 & $\begin{array}{c}0,89 \\
(0,67-1,17)\end{array}$ & & $\begin{array}{c}0,87 \\
(0,66-1,15)\end{array}$ & \\
\hline \multicolumn{11}{|l|}{ Nível 2} \\
\hline Atividade profissional na UBS & & & $<0,001$ & & 0,01 & & & $<0,001$ & & 0,39 \\
\hline Agentes comunitários de saúde & 75,9 & 1,00 & & 1,00 & & 81,6 & 1,00 & & 1,00 & \\
\hline Auxiliar/Técnico de enfermagem e & 72,9 & 0,64 & & 0,85 & & 47,1 & 0,58 & & 0,75 & \\
\hline Auxiliar/Técnico de consultório dentário & & $(0,57-0,72)$ & & $(0,72-1,00)$ & & & $(0,52-0,64)$ & & $(0,65-0,86)$ & \\
\hline Médico & 65,9 & $\begin{array}{c}0,48 \\
(0,41-0,57)\end{array}$ & & $\begin{array}{c}0,80 \\
(0,61-1,04)\end{array}$ & & 44,4 & $\begin{array}{c}0,54 \\
(0,47-0,63)\end{array}$ & & $\begin{array}{c}0,85 \\
(0,70-1,02)\end{array}$ & \\
\hline Enfermeiro & 60,5 & $\begin{array}{c}0,86 \\
(0,76-0,98)\end{array}$ & & $\begin{array}{c}1,04 \\
(0,85-1,28)\end{array}$ & & 71,4 & $\begin{array}{c}0,88 \\
(0,79-0,97)\end{array}$ & & $\begin{array}{c}0,99 \\
(0,86-1,14)\end{array}$ & \\
\hline Odontólogo & 58,2 & $\begin{array}{c}0,87 \\
(0,75-1,04)\end{array}$ & & $\begin{array}{c}1,45 \\
(1,13-1,87)\end{array}$ & & 62,9 & $\begin{array}{c}0,77 \\
(0,68-0,88)\end{array}$ & & $\begin{array}{c}1,17 \\
(0,99-1,38)\end{array}$ & \\
\hline Outros de nível superior & 51,1 & $\begin{array}{c}0,82 \\
(0,64-1,05)\end{array}$ & & $\begin{array}{c}1,40 \\
(0,98-2,00)\end{array}$ & & 39,8 & $\begin{array}{c}0,49 \\
(0,39-0,61)\end{array}$ & & $\begin{array}{c}0,68 \\
(0,50-0,92)\end{array}$ & \\
\hline Regime de trabalho precarizado & & & 0,003 & & 0,71 & & & $<0,001$ & & 0,78 \\
\hline Não & 55,6 & 1,00 & & 1,00 & & 57,0 & 1,00 & & 1,00 & \\
\hline Sim & 64,4 & $\begin{array}{c}1,16 \\
(1,05-1,27)\end{array}$ & & $\begin{array}{c}1,02 \\
(0,91-1,14)\end{array}$ & & 75,0 & $\begin{array}{c}1,32 \\
(1,24-1,40)\end{array}$ & & $\begin{array}{c}0,99 \\
(0,92-1,06)\end{array}$ & \\
\hline Vinculação ao plano de carreira & & & 0,007 & & 0,02 & & & $<0,001$ & & $<0,001$ \\
\hline Não & 60,4 & 1,00 & & 1,00 & & 70,5 & 1,00 & & 1,00 & \\
\hline Sim & 48,2 & $\begin{array}{c}0,80 \\
(0,68-0,94)\end{array}$ & & $\begin{array}{c}0,81 \\
(0,67-0,97)\end{array}$ & & 47,8 & $\begin{array}{c}0,68 \\
(0,60-0,77)\end{array}$ & & $\begin{array}{c}0,77 \\
(0,68-0,88)\end{array}$ & \\
\hline
\end{tabular}

(continua) 
Tabela 3 (continuação)

\begin{tabular}{|c|c|c|c|c|c|c|c|c|c|c|}
\hline \multirow[t]{3}{*}{ Níveis/Variáveis } & \multicolumn{5}{|c|}{ Região Sul } & \multicolumn{5}{|c|}{ Região Nordeste } \\
\hline & \multicolumn{3}{|c|}{ Análise bruta } & \multicolumn{2}{|c|}{ Análise ajustada } & \multicolumn{3}{|c|}{ Análise bruta } & \multicolumn{2}{|c|}{ Análise ajustada } \\
\hline & $\%$ & $\begin{array}{c}\text { RP } \\
\text { (IC95\%) }\end{array}$ & $\begin{array}{l}\text { Valor } \\
\text { de } p\end{array}$ & $\begin{array}{c}\text { RP } \\
\text { (IC95\%) }\end{array}$ & $\begin{array}{l}\text { Valor } \\
\text { de } p\end{array}$ & $\%$ & $\begin{array}{c}\mathrm{RP} \\
\text { (IC95\%) }\end{array}$ & $\begin{array}{l}\text { Valor } \\
\text { de } p\end{array}$ & $\begin{array}{c}\text { RP } \\
\text { (IC95\%) }\end{array}$ & $\begin{array}{l}\text { Valor } \\
\text { de p }\end{array}$ \\
\hline Primeiro emprego & & & 0,17 & & 0,94 & & & 0,76 & & 0,26 \\
\hline Não & 58,5 & 1,00 & & 1,00 & & 66,1 & 1,00 & & 1,00 & \\
\hline \multirow[t]{2}{*}{ Sim } & 53,3 & 0,91 & & 0,99 & & 65,4 & 0,99 & & 0,96 & \\
\hline & & $(0,80-1,04)$ & & $(0,86-1,15)$ & & & $(0,93-1,06)$ & & $(0,89-1,03)$ & \\
\hline Outro emprego além do atual na UBS & & & $<0,001$ & & 0,04 & & & $<0,001$ & & $<0,001$ \\
\hline Não & 63,2 & 1,00 & & 1,00 & & 72,6 & 1,00 & & 1,00 & \\
\hline \multirow[t]{2}{*}{$\operatorname{Sim}$} & 39,6 & 0,63 & & 0,83 & & 45,2 & 0,62 & & 0,80 & \\
\hline & & $(0,54-0,73)$ & & $(0,69-0,99)$ & & & $(0,56-0,69)$ & & $(0,72-0,89)$ & \\
\hline Tempo de atuação na UBS em tercis & & & $<0,001$ * & & 0,25 * & & & 0,10 * & & 0,27 * \\
\hline Até 24 meses & 62,3 & 1,00 & & 1,00 & & 64,8 & 1,00 & & 1,00 & \\
\hline \multirow[t]{2}{*}{ 25-108 meses } & 52,5 & 0,84 & & 0,97 & & 72,5 & 1,12 & & 1,07 & \\
\hline & & $(0,76-0,94)$ & & $(0,87-1,08)$ & & & $(1,05-1,19)$ & & $(1,00-1,15)$ & \\
\hline \multirow[t]{2}{*}{109 meses ou mais } & 42,0 & 0,67 & & 0,84 & & 50,4 & 0,78 & & 1,02 & \\
\hline & & $(0,53-0,85)$ & & $(0,63-1,12)$ & & & $(0,68-0,89)$ & & $(0,89-1,17)$ & \\
\hline $\begin{array}{l}\text { Capacitação profissional em saúde da } \\
\text { mulher }\end{array}$ & & & $<0,001$ & & 0,45 & & & $<0,001$ & & 0,96 \\
\hline Não & 46,0 & 1,00 & & 1,00 & & 56,0 & 1,00 & & 1,00 & \\
\hline \multirow[t]{2}{*}{$\operatorname{Sim}$} & 72,1 & 1,56 & & 0,91 & & 76,7 & 1,37 & & 1,00 & \\
\hline & & $(1,39-1,76)$ & & $(0,72-1,16)$ & & & $(1,27-1,47)$ & & $(0,90-1,12)$ & \\
\hline $\begin{array}{l}\text { Capacitação profissional em saúde da } \\
\text { criança }\end{array}$ & & & $<0,001$ & & 0,04 & & & $<0,001$ & & 0,46 \\
\hline Não & 46,5 & 1,00 & & 1,00 & & 54,2 & 1,00 & & 1,00 & \\
\hline \multirow[t]{2}{*}{ Sim } & 72,5 & 1,56 & & 1,20 & & 77,8 & 1,43 & & 1,03 & \\
\hline & & $(1,34-1,75)$ & & $(1,01-1,42)$ & & & $(1,33-1,55)$ & & $(0,95-1,13)$ & \\
\hline Capacitação profissional no manejo de & & & $<0,001$ & & 0,002 & & & $<0,001$ & & $<0,001$ \\
\hline \multicolumn{11}{|l|}{ doenças crônicas } \\
\hline Não & 44,2 & 1,00 & & 1,00 & & 52,2 & 1,00 & & 1,00 & \\
\hline \multirow[t]{2}{*}{ Sim } & 72,6 & 1,64 & & 1,33 & & 77,4 & 1,48 & & 1,29 & \\
\hline & & $(1,46-1,85)$ & & $(1,11-1,59)$ & & & $(1,37-1,61)$ & & $(1,19-1,39)$ & \\
\hline Utilização de protocolos pelo profissional & & & $<0,001$ & & 0,05 & & & $<0,001$ & & $<0,001$ \\
\hline Não & 54,3 & 1,00 & & 1,00 & & 58,9 & 1,00 & & 1,00 & \\
\hline \multirow[t]{2}{*}{$\operatorname{Sim}$} & 63,2 & 1,16 & & 1,11 & & 71,9 & 1,22 & & 1,20 & \\
\hline & & $(1,06-1,28)$ & & $(1,00-1,22)$ & & & $(1,15-1,30)$ & & $(1,12-1,28)$ & \\
\hline Satisfação com as reuniões comunitárias & & & $<0,001 *$ & & $<0,001 *$ & & & $<0,001 *$ & & 0,08 * \\
\hline \multicolumn{11}{|l|}{ notas $0-10$ em tercis } \\
\hline $0-4,0$ & 44,2 & 1,00 & & 1,00 & & 61,3 & 1,00 & & 1,00 & \\
\hline \multirow[t]{2}{*}{$4,1-7,0$} & 63,0 & 1,43 & & 1,38 & & 74,4 & 1,21 & & 1,09 & \\
\hline & & $(1,25-1,62)$ & & $(1,18-1,62)$ & & & $(1,13-1,31)$ & & $(1,01-1,18)$ & \\
\hline \multirow[t]{2}{*}{$7,1-10,0$} & 76,6 & 1,73 & & 1,57 & & 76,1 & 1,24 & & 1,07 & \\
\hline & & $(1,53-1,96)$ & & $(1,35-1,82)$ & & & $(1,15-1,34)$ & & $(0,99-1,17)$ & \\
\hline Vinculação da UBS com ensino & & & 0,02 & & 0,05 & & & $<0,001$ & & $<0,001$ \\
\hline Não & 60,1 & 1,00 & & 1,00 & & 60,8 & 1,00 & & 1,00 & \\
\hline \multirow[t]{2}{*}{ Sim } & 54,3 & 0,89 & & 0,91 & & 70,9 & 1,17 & & 1,21 & \\
\hline & & $(0,81-0,98)$ & & $(0,82-1,00)$ & & & $(1,10-1,24)$ & & $(1,13-1,29)$ & \\
\hline
\end{tabular}

(continua) 
Tabela 3 (continuação)

\begin{tabular}{|c|c|c|c|c|c|c|c|c|c|c|}
\hline \multirow[t]{3}{*}{ Níveis/Variáveis } & \multicolumn{5}{|c|}{ Região Sul } & \multicolumn{5}{|c|}{ Região Nordeste } \\
\hline & \multicolumn{3}{|c|}{ Análise bruta } & \multicolumn{2}{|c|}{ Análise ajustada } & \multicolumn{3}{|c|}{ Análise bruta } & \multicolumn{2}{|c|}{ Análise ajustada } \\
\hline & $\%$ & $\begin{array}{c}\text { RP } \\
\text { (IC95\%) }\end{array}$ & $\begin{array}{l}\text { Valor } \\
\text { de } p\end{array}$ & $\begin{array}{c}\text { RP } \\
\text { (IC95\%) }\end{array}$ & $\begin{array}{l}\text { Valor } \\
\text { de p }\end{array}$ & $\%$ & $\begin{array}{c}\text { RP } \\
\text { (IC95\%) }\end{array}$ & $\begin{array}{l}\text { Valor } \\
\text { de } p\end{array}$ & $\begin{array}{c}\text { RP } \\
\text { (IC95\%) }\end{array}$ & $\begin{array}{l}\text { Valor } \\
\text { de } p\end{array}$ \\
\hline \multicolumn{11}{|l|}{ Nível 3} \\
\hline Satisfação com a saúde & & & 0,02 * & & 0,09 * & & & 0,84 * & & 1,00 * \\
\hline Insatisfeito & 50,3 & 1,00 & & 1,00 & & 63,9 & 1,00 & & 1,00 & \\
\hline \multirow[t]{2}{*}{ Indiferente } & 55,5 & 1,10 & & 1,06 & & 68,3 & 1,07 & & 1,00 & \\
\hline & & $(0,92-1,32)$ & & $(0,87-1,30)$ & & & $(0,95-1,21)$ & & $(0,88-1,13)$ & \\
\hline \multirow[t]{2}{*}{ Satisfeito } & 59,8 & 1,19 & & 1,14 & & 65,5 & 1,03 & & 1,00 & \\
\hline & & $(1,01-1,40)$ & & $(0,95-1,38)$ & & & $(0,92-1,15)$ & & $(0,88-1,13)$ & \\
\hline Transtornos mentais comuns (SRQ-20) & & & 0,73 & & 0,28 & & & 0,62 & & 0,65 \\
\hline Negativo & 57,2 & 1,00 & & 1,00 & & 65,5 & 1,00 & & 1,00 & \\
\hline \multirow[t]{2}{*}{ Positivo } & 58,5 & 1,02 & & 1,09 & & 66,9 & 1,02 & & 1,02 & \\
\hline & & $(0,90-1,17)$ & & $(0,94-1,26)$ & & & $(0,94-1,11)$ & & $(0,94-1,11)$ & \\
\hline
\end{tabular}

IC95\%: intervalo de 95\% de confiança; RP: razão de prevalência; SRQ-20: Self-Report Questionnaire-20.

* Teste de Wald para tendência.

\section{Tabela 4}

Prevalência de atividades na comunidade, análise bruta e ajustada da amostra de trabalhadores das unidades básicas de saúde (UBS) conforme o modelo de atenção. Brasil, 2005.

\begin{tabular}{|c|c|c|c|c|c|c|c|c|c|c|}
\hline \multirow[t]{3}{*}{ Níveis/Variáveis } & \multicolumn{5}{|c|}{ PSF } & \multicolumn{5}{|c|}{ Tradicional } \\
\hline & \multicolumn{3}{|c|}{ Análise bruta } & \multicolumn{2}{|c|}{ Análise ajustada } & \multicolumn{3}{|c|}{ Análise bruta } & \multicolumn{2}{|c|}{ Análise ajustada } \\
\hline & $\%$ & $\begin{array}{c}\text { RP } \\
\text { (IC95\%) }\end{array}$ & $\begin{array}{l}\text { Valor } \\
\text { de } p\end{array}$ & $\begin{array}{c}\text { RP } \\
\text { (IC95\%) }\end{array}$ & $\begin{array}{l}\text { Valor } \\
\text { de } p\end{array}$ & $\%$ & $\begin{array}{c}\text { RP } \\
\text { (IC95\%) }\end{array}$ & $\begin{array}{l}\text { Valor } \\
\text { de } p\end{array}$ & $\begin{array}{c}\text { RP } \\
\text { (IC95\%) }\end{array}$ & $\begin{array}{l}\text { Valor } \\
\text { de } p\end{array}$ \\
\hline \multicolumn{11}{|l|}{ Nível 1} \\
\hline Sexo & & & 0,29 & & 0,44 & & & 0,001 & & 0,09 \\
\hline Masculino & 73,2 & 1,00 & & 1,00 & & 27,3 & 1,00 & & 1,00 & \\
\hline \multirow[t]{2}{*}{ Feminino } & 76,0 & 1,04 & & 1,03 & & 40,4 & 1,48 & & 1,22 & \\
\hline & & $(0,97-1,11)$ & & $(0,96-1,10)$ & & & $(1,18-1,85)$ & & $(0,97-1,52)$ & \\
\hline Idade (anos) & & & 0,97 * & & 0,86 * & & & $<0,001$ * & & $<0,001$ * \\
\hline $16-29$ & 75,0 & 1,00 & & 1,00 & & 48,9 & 1,00 & & 1,00 & \\
\hline \multirow[t]{2}{*}{$30-49$} & 75,8 & 1,01 & & 1,01 & & 38,8 & 0,79 & & 0,81 & \\
\hline & & $(0,96-1,06)$ & & $(0,96-1,07)$ & & & $(0,68-0,93)$ & & $(0,70-0,95)$ & \\
\hline \multirow[t]{2}{*}{50 ou mais } & 74,3 & 0,99 & & 1,00 & & 22,9 & 0,47 & & 0,51 & \\
\hline & & $(0,91-1,08)$ & & $(0,91-1,09)$ & & & $(0,35-0,62)$ & & $(0,38-0,68)$ & \\
\hline Escolaridade & & & 0,07 * & & 0,07 * & & & $<0,001$ * & & $<0,001$ * \\
\hline Ensino Fundamental incompleto & 81,4 & 1,00 & & 1,00 & & 37,5 & 1,00 & & 1,00 & \\
\hline \multirow[t]{2}{*}{ Ensino Fundamental completo } & 78,8 & 0,97 & & 0,97 & & 57,6 & 1,54 & & 1,37 & \\
\hline & & $(0,82-1,15)$ & & $(0,82-1,15)$ & & & $(0,76-3,08)$ & & $(0,71-2,64)$ & \\
\hline \multirow[t]{2}{*}{ Ensino Médio incompleto } & 78,1 & 0,96 & & 0,96 & & 47,4 & 1,26 & & 1,11 & \\
\hline & & $(0,82-1,13)$ & & $(0,82-1,13)$ & & & $(0,63-2,52)$ & & $(0,58-2,14)$ & \\
\hline \multirow[t]{2}{*}{ Ensino Médio completo } & 76,1 & 0,93 & & 0,93 & & 47,8 & 1,28 & & 1,04 & \\
\hline & & $(0,81-1,08)$ & & $(0,81-1,08)$ & & & $(0,67-2,42)$ & & $(0,57-1,92)$ & \\
\hline \multirow[t]{2}{*}{ Ensino Superior incompleto } & 72,5 & 0,89 & & 0,89 & & 43,7 & 1,16 & & 0,90 & \\
\hline & & $(0,75-1,06)$ & & $(0,75-1,06)$ & & & $(0,59-2,31)$ & & $(0,46-1,74)$ & \\
\hline \multirow[t]{2}{*}{ Ensino Superior completo } & 74,2 & 0,91 & & 0,91 & & 26,0 & 0,69 & & 0,63 & \\
\hline & & $(0,79-1,06)$ & & $(0,79-1,06)$ & & & $(0,36-1,33)$ & & $(0,34-1,17)$ & \\
\hline
\end{tabular}

(continua) 


\begin{tabular}{|c|c|c|c|c|c|c|c|c|c|c|}
\hline \multirow[t]{3}{*}{ Níveis/Variáveis } & \multicolumn{5}{|c|}{ PSF } & \multicolumn{5}{|c|}{ Tradicional } \\
\hline & \multicolumn{3}{|c|}{ Análise bruta } & \multicolumn{2}{|c|}{ Análise ajustada } & \multicolumn{3}{|c|}{ Análise bruta } & \multicolumn{2}{|c|}{ Análise ajustada } \\
\hline & $\%$ & $\begin{array}{c}\text { RP } \\
\text { (IC95\%) }\end{array}$ & $\begin{array}{l}\text { Valor } \\
\text { de } p\end{array}$ & $\begin{array}{c}\text { RP } \\
\text { (IC95\%) }\end{array}$ & $\begin{array}{l}\text { Valor } \\
\text { de } p\end{array}$ & $\%$ & $\begin{array}{c}\text { RP } \\
\text { (IC95\%) }\end{array}$ & $\begin{array}{l}\text { Valor } \\
\text { de } p\end{array}$ & $\begin{array}{c}\text { RP } \\
\text { (IC95\%) }\end{array}$ & $\begin{array}{l}\text { Valor } \\
\text { de } p\end{array}$ \\
\hline \multicolumn{11}{|l|}{ Nível 2} \\
\hline Atividade profissional na UBS & & & 0,07 & & 0,02 & & & $<0,001$ & & 0,46 \\
\hline Agentes comunitários de saúde & 80,9 & 1,00 & & 1,00 & & 74,2 & 1,00 & & 1,00 & \\
\hline Auxiliar/Técnico de enfermagem e & 65,6 & 0,81 & & 0,90 & & 19,4 & 0,26 & & 0,39 & \\
\hline Auxiliar/Técnico de consultório dentário & & $(0,76-0,87)$ & & $(0,81-0,98)$ & & & $(0,21-0,33)$ & & $(0,27-0,57)$ & \\
\hline \multirow[t]{2}{*}{ Médico } & 64,8 & 0,80 & & 0,87 & & 12,6 & 0,17 & & 0,26 & \\
\hline & & $(0,73-0,88)$ & & $(0,76-1,00)$ & & & $(0,12-0,24)$ & & $(0,13-0,52)$ & \\
\hline \multirow[t]{2}{*}{ Enfermeiro } & 77,2 & 0,95 & & 0,99 & & 44,7 & 0,60 & & 0,68 & \\
\hline & & $(0,89-1,03)$ & & $(0,88-1,10)$ & & & $(0,48-0,76)$ & & $(0,46-1,00)$ & \\
\hline \multirow[t]{2}{*}{ Odontólogo } & 87,7 & 1,08 & & 1,28 & & 27,8 & 0,37 & & 0,57 & \\
\hline & & $(1,01-1,16)$ & & $(1,15-1,43)$ & & & $(0,27-0,53)$ & & $(0,30-1,08)$ & \\
\hline \multirow[t]{2}{*}{ Outros de nível superior } & 66,1 & 0,82 & & 0,99 & & 34,0 & 0,46 & & 0,60 & \\
\hline & & $(0,66-0,99)$ & & $(0,80-1,24)$ & & & $(0,35-0,60)$ & & $(0,36-1,00)$ & \\
\hline Regime de trabalho precarizado & & & 0,003 & & 0,72 & & & $<0,001$ & & 0,95 \\
\hline Não & 73,0 & 1,00 & & 1,00 & & 28,0 & 1,00 & & 1,00 & \\
\hline \multirow[t]{2}{*}{$\operatorname{Sim}$} & 78,4 & 1,07 & & 1,01 & & 56,4 & 2,02 & & 0,99 & \\
\hline & & $(1,03-1,13)$ & & $(0,96-1,07)$ & & & $(1,75-2,33)$ & & $(0,82-1,21)$ & \\
\hline Vinculação ao plano de carreira & & & 0,30 & & 0,07 & & & $<0,001$ & & 0,03 \\
\hline Não & 76,2 & 1,00 & & 1,00 & & 44,0 & 1,00 & & 1,00 & \\
\hline \multirow[t]{2}{*}{$\operatorname{Sim}$} & 72,9 & 0,96 & & 0,92 & & 23,7 & 0,54 & & 0,69 & \\
\hline & & $(0,88-1,04)$ & & $(0,83-1,01)$ & & & $(0,42-0,69)$ & & $(0,49-0,97)$ & \\
\hline Primeiro emprego & & & 0,23 & & 0,49 & & & 0,13 & & 0,13 \\
\hline Não & 74,9 & 1,00 & & 1,00 & & 38,8 & 1,00 & & 1,00 & \\
\hline \multirow[t]{2}{*}{ Sim } & 77,3 & 1,03 & & 1,02 & & 33,8 & 0,87 & & 0,84 & \\
\hline & & $(0,98-1,09)$ & & $(0,96-1,09)$ & & & $(0,73-1,04)$ & & $(0,66-1,05)$ & \\
\hline Outro emprego além do atual na UBS & & & $<0,001$ & & 0,13 & & & $<0,001$ & & $<0,001$ \\
\hline Não & 77,1 & 1,00 & & 1,00 & & 48,6 & 1,00 & & 1,00 & \\
\hline \multirow[t]{2}{*}{ Sim } & 68,1 & 0,88 & & 0,94 & & 16,8 & 0,35 & & 0,44 & \\
\hline & & $(0,82-0,95)$ & & $(0,87-1,02)$ & & & $(0,27-0,43)$ & & $(0,30-0,63)$ & \\
\hline Tempo de atuação na UBS em tercis & & & 0,007 * & & $<0,001 *$ & & & 0,46 * & & 0,94 * \\
\hline Até 24 meses & 73,5 & 1,00 & & 1,00 & & 35,8 & 1,00 & & 1,00 & \\
\hline \multirow[t]{2}{*}{$25-108$ meses } & 78,6 & 1,07 & & 1,08 & & 43,2 & 1,20 & & 1,04 & \\
\hline & & $(1,02-1,12)$ & & $(1,02-1,14)$ & & & $(1,02-1,42)$ & & $(0,85-1,28)$ & \\
\hline \multirow[t]{2}{*}{109 meses ou mais } & 78,6 & 1,07 & & 1,15 & & 30,0 & 0,83 & & 0,98 & \\
\hline & & $(0,97-1,18)$ & & $(1,04-1,28)$ & & & $(0,66-1,06)$ & & $(0,71-1,36)$ & \\
\hline \multicolumn{11}{|l|}{ mulher } \\
\hline Não & 69,5 & 1,00 & & 1,00 & & 23,0 & 1,00 & & 1,00 & \\
\hline \multirow[t]{2}{*}{$\operatorname{Sim}$} & 81,6 & 1,17 & & 0,98 & & 57,1 & 2,48 & & 1,35 & \\
\hline & & $(1,11-1,24)$ & & $(0,91-1,05)$ & & & $(2,04-3,02)$ & & $(1,00-1,83)$ & \\
\hline Capacitação profissional em saúde da & & & $<0,001$ & & 0,90 & & & $<0,001$ & & 0,71 \\
\hline criança & & & & & & & & & & \\
\hline Não & 69,0 & 1,00 & & 1,00 & & 22,7 & 1,00 & & 1,00 & \\
\hline Sim & 80,0 & 1,17 & & 1,01 & & 62,0 & 2,73 & & 1,07 & \\
\hline & & $(1,10-1,24)$ & & $(0,91-1,11)$ & & & $(2,26-3,31)$ & & $(0,74-1,56)$ & \\
\hline
\end{tabular}

(continua) 


\begin{tabular}{|c|c|c|c|c|c|c|c|c|c|c|}
\hline \multirow[t]{3}{*}{ Níveis/Variáveis } & \multicolumn{5}{|c|}{ PSF } & \multicolumn{5}{|c|}{ Tradicional } \\
\hline & \multicolumn{3}{|c|}{ Análise bruta } & \multicolumn{2}{|c|}{ Análise ajustada } & \multicolumn{3}{|c|}{ Análise bruta } & \multicolumn{2}{|c|}{ Análise ajustada } \\
\hline & $\%$ & $\begin{array}{c}\text { RP } \\
\text { (IC95\%) }\end{array}$ & $\begin{array}{l}\text { Valor } \\
\text { de } p\end{array}$ & $\begin{array}{c}\mathrm{RP} \\
\text { (IC95\%) }\end{array}$ & $\begin{array}{l}\text { Valor } \\
\text { de p }\end{array}$ & $\%$ & $\begin{array}{c}\mathrm{RP} \\
\text { (IC95\%) }\end{array}$ & $\begin{array}{l}\text { Valor } \\
\text { de } p\end{array}$ & $\begin{array}{c}\mathrm{RP} \\
\text { (IC95\%) }\end{array}$ & $\begin{array}{l}\text { Valor } \\
\text { de p }\end{array}$ \\
\hline $\begin{array}{l}\text { Capacitação profissional no manejo de } \\
\text { doenças crônicas }\end{array}$ & & & $<0,001$ & & $<0,001$ & & & $<0,001$ & & 0,001 \\
\hline Não & 65,8 & 1,00 & & 1,00 & & 22,4 & 1,00 & & 1,00 & \\
\hline Sim & 82,0 & $\begin{array}{c}1,25 \\
(1,17-1,32)\end{array}$ & & $\begin{array}{c}1,19 \\
(1,12-1,27)\end{array}$ & & 57,9 & $\begin{array}{c}2,58 \\
(2,12-3,14)\end{array}$ & & $\begin{array}{c}1,66 \\
(1,23-2,23)\end{array}$ & \\
\hline Utilização de protocolos pelo profissional & & & $<0,001$ & & $<0,001$ & & & $<0,001$ & & 0,002 \\
\hline Não & 70,8 & 1,00 & & 1,00 & & 32,3 & 1,00 & & 1,00 & \\
\hline $\operatorname{Sim}$ & 80,4 & $\begin{array}{c}1,14 \\
(1,08-1,19)\end{array}$ & & $\begin{array}{c}1,14 \\
(1,08-1,20)\end{array}$ & & 43,3 & $\begin{array}{c}1,34 \\
(1,15-1,56)\end{array}$ & & $\begin{array}{c}1,31 \\
(1,11-1,56)\end{array}$ & \\
\hline $\begin{array}{l}\text { Satisfação com as reuniões comunitárias } \\
\text { notas } 0-10 \text { em tercis }\end{array}$ & & & $<0,001$ * & & 0,002 * & & & $<0,001$ * & & 0,94 * \\
\hline $0-4,0$ & 69,8 & 1,00 & & 1,00 & & 34,8 & 1,00 & & 1,00 & \\
\hline $4,1-7,0$ & 77,1 & $\begin{array}{c}1,10 \\
(1,04-1,18)\end{array}$ & & $\begin{array}{c}1,04 \\
(0,97-1,12)\end{array}$ & & 49,2 & $\begin{array}{c}1,41 \\
(1,19-1,67)\end{array}$ & & $\begin{array}{c}1,18 \\
(0,97-1,44)\end{array}$ & \\
\hline $7,1-10,0$ & 82,4 & $\begin{array}{c}1,18 \\
(1,11-1,26)\end{array}$ & & $\begin{array}{c}1,11 \\
(1,04-1,19)\end{array}$ & & 51,4 & $\begin{array}{c}1,46 \\
(1,22-1,78)\end{array}$ & & $\begin{array}{c}0,97 \\
(0,76-1,23)\end{array}$ & \\
\hline Vinculação da UBS com ensino & & & 0,009 & & 0,80 & & & 0,49 & & 0,001 \\
\hline Não & 78,1 & 1,00 & & 1,00 & & 38,5 & 1,00 & & 1,00 & \\
\hline Sim & 73,4 & $\begin{array}{c}0,94 \\
(0,90-0,98)\end{array}$ & & $\begin{array}{c}0,99 \\
(0,94-1,05)\end{array}$ & & 36,5 & $\begin{array}{c}0,95 \\
(0,81-1,11)\end{array}$ & & $\begin{array}{c}1,32 \\
(1,12-1,56)\end{array}$ & \\
\hline Nível 3 & & & & & & & & & & \\
\hline Satisfação com a saúde & & & 0,001 * & & 0,005 * & & & 0,57 * & & 0,94 * \\
\hline Insatisfeito & 68,9 & 1,00 & & 1,00 & & 35,8 & 1,00 & & 1,00 & \\
\hline Indiferente & 72,4 & $\begin{array}{c}1,05 \\
(0,95-1,16)\end{array}$ & & $\begin{array}{c}1,10 \\
(0,98-1,24)\end{array}$ & & 41,9 & $\begin{array}{c}1,17 \\
(0,89-1,55)\end{array}$ & & $\begin{array}{c}0,95 \\
(0,70-1,29)\end{array}$ & \\
\hline Satisfeito & 77,7 & $\begin{array}{c}1,13 \\
(1,03-1,23)\end{array}$ & & $\begin{array}{c}1,15 \\
(1,04-1,29)\end{array}$ & & 36,5 & $\begin{array}{c}1,02 \\
(0,79-1,31)\end{array}$ & & $\begin{array}{c}0,97 \\
(0,73-1,30)\end{array}$ & \\
\hline Transtornos mentais comuns (SRQ-20) & & & 0,09 & & 0,97 & & & 0,008 & & 0,53 \\
\hline Negativo & 75,9 & 1,00 & & 1,00 & & 36,0 & 1,00 & & 1,00 & \\
\hline Positivo & 71,4 & $\begin{array}{c}0,94 \\
(0,88-1,01)\end{array}$ & & $\begin{array}{c}1,00 \\
(0,92-1,09)\end{array}$ & & 46,5 & $\begin{array}{c}1,29 \\
(1,07-1,56)\end{array}$ & & $\begin{array}{c}1,07 \\
(0,87-1,31)\end{array}$ & \\
\hline
\end{tabular}

IC95\%: intervalo de 95\% de confiança; PSF: Programa Saúde da Família; RP: razão de prevalência; SRQ-20: Self-Report Questionnaire-20.

* Teste de Wald para tendência.

tradicional $(37,7 \%)$ e o PSF $(75,5 \%)$. Considerando-se que entre as atribuições comuns a todos os profissionais das equipes de saúde da família está o cuidado em saúde da população adscrita, prioritariamente no âmbito da unidade de saúde, no domicílio e nos demais espaços comunitários ${ }^{1}$, esperar-se-ia uma prevalência ainda maior no PSF.

Estudos apontam que embora as equipes de saúde se esforcem em realizar atividades de caráter coletivo, ainda é baixa a participação dos trabalhadores em grupos e instituições locais com vistas à resolução dos problemas da população. Isso evidencia a escassez de atividades participativas com a comunidade e indica que os profissionais das equipes de saúde utilizam grande parte do seu tempo atendendo à demanda espontânea 8,12,15,25. Alguns fatores como o despreparo da gestão, que ainda reitera a lógica quantitativa de produção, a priorização do atendimento à demanda e a inadequação da formação e capacitação profissional podem dificultar a inserção dos trabalhadores em práticas na comunidade. Especificamente quanto à formação tem sido constatado ainda que o perfil dos profissionais egressos dos cursos não é adequado o suficiente para uma atuação integral 10,13,26. 
A prática de saúde mais realizada na comunidade, independentemente da região ou modelo de atenção, foi a educação em saúde em datas festivas, palestras e grupos, sendo referida por $67 \%$ dos trabalhadores, corroborando as evidências sobre o tema que apontam as atividades extramuros de promoção da saúde e prevenção de doenças como as mais incorporadas pelas equipes 14,27. Entre os demais tipos de práticas foi evidenciado o escasso envolvimento dos trabalhadores junto ao controle social, contrariando, portanto, o referencial normativo do SUS 28,29,30. $\mathrm{Na}$ atualidade, apesar de encontrarmos Conselhos Municipais de Saúde constituídos na maioria dos municípios brasileiros, a sua capacidade de exercer o controle social ainda é baixa 29 .

No presente estudo encontrou-se que a exposição mais favorecedora da realização de práticas profissionais na comunidade foi a capacitação no manejo de doenças crônicas: hipertensão arterial sistêmica e diabetes mellitus. Os trabalhadores que fizeram essa capacitação tiveram uma probabilidade $30 \%$ maior para a realização de práticas na comunidade. Esse achado pode ser explicado devido à importância epidemiológica dessas doenças e pelas políticas públicas nacionais implementadas para seu adequado controle, sendo uma das estratégias a ampliação da capacitação dos profissionais de saúde 27,31. Dentre as medidas necessárias para o enfrentamento da hipertensão arterial e do diabetes mellitus estão a formação de grupos de educação em saúde e a promoção de caminhadas coletivas 14,32,33.

Outro fator associado ao desfecho foi a utilização de protocolo pelos trabalhadores de saúde para suas atividades profissionais. A estruturação dos programas de saúde em atenção básica à saúde tradicionalmente envolve a produção e divulgação de protocolos clínicos aos serviços de saúde. O conteúdo de tais documentos visa, entre outros aspectos, a estimular a adoção de práticas de promoção à saúde e de prevenção como estratégias de controle dos agravos à saúde. Em um estudo realizado na Espanha, Puig Ribeira et al. 34 mostraram que profissionais que usam protocolos se diferenciam dos demais em relação ao incentivo de estilos de vida saudáveis. Essas diferenças são principalmente devidas ao conhecimento adquirido e ao reconhecimento da importância do tema para a saúde dos seus pacientes e de si próprio.

A satisfação com as reuniões comunitárias também se associou à maior prevalência de práticas de saúde na comunidade. As reuniões comunitárias são o espaço onde os trabalhadores de saúde procuram estimular a participação social por meio de momentos de discussão junto às famílias e aos grupos da comunidade. Também visam à construção da cidadania, à transformação dos sujeitos sociais em sujeitos políticos e ao estabelecimento de vínculos de co-responsabilidade 17,31. A satisfação dos trabalhadores com as reuniões pode expressar real envolvimento com a comunidade no levantamento de seus problemas e planejamento de ações em conjunto com a equipe.

De acordo com a função na UBS, neste estudo os trabalhadores com maior probabilidade de sair do âmbito do serviço para realizar práticas de saúde na comunidade foram os odontólogos do PSF do Sul do país. Embora as atividades curativas ainda tenham maior prevalência na prática dos odontólogos, a valorização das ações preventivas e de educação em saúde bucal se faz presente entre as equipes na atualidade 35 . Destaca-se ainda, que a maior participação dos odontólogos em práticas na comunidade quando comparados aos demais profissionais, pode ser um resultado positivo em favor da reorganização da atenção em saúde bucal na atenção básica à saúde preconizada pela Política Brasileira de Saúde Bucal 36.

A diferença encontrada quanto ao sexo, indicando maior realização de práticas profissionais na comunidade pelas mulheres, pode ser explicada pela tendência atual da feminilização da força de trabalho em saúde no Brasil 8,10,13,27,37.

Os trabalhadores de UBS vinculadas a instituições de ensino das regiões Sul e Nordeste e aqueles das UBS tradicionais tiveram maior freqüência de realização de práticas de saúde na comunidade. Esse achado pode ser um indicador do efetivo comprometimento das instituições de ensino com o desenvolvimento de recursos humanos para a atenção básica 10 .

Em relação ao tempo de atuação na UBS, acredita-se que a associação encontrada entre maior tempo de atuação na UBS e probabilidade maior de realização de práticas na comunidade se deva à formação de vínculos entre profissional e comunidade e o comprometimento com a população. Estudos referem que a alta rotatividade das equipes, especialmente no PSF, impede a formação de vínculos e o desenvolvimento de novas práticas profissionais, voltadas para família e comunidade 10,31,37.

A associação positiva encontrada entre realização de práticas na comunidade e a satisfação do trabalhador com a sua saúde parece consistente com a literatura científica, que mostra que os mais satisfeitos são mais ativos, mais interessados na sua saúde e na saúde de seus pacientes 34 .

Os resultados do estudo mostram que é necessário ampliar as ações das equipes junto à comunidade, particularmente aquelas associadas ao controle e ações sociais, considerando que a atenção básica à saúde é o nível do sistema de 
saúde que possibilita a construção de práticas que efetivamente respondem às demandas da sociedade. Embora no PSF a participação dos trabalhadores em práticas na comunidade seja maior, ainda está distante das expectativas.
É preciso ainda estimular e institucionalizar a participação da comunidade nas políticas públicas, visando prioritariamente à elaboração de um plano local de enfrentamento dos determinantes do processo saúde/doença.

\section{Resumo}

Com o objetivo de verificar a prevalência de participação em práticas de saúde na comunidade, foi realizado um estudo transversal com 3.743 trabalhadores da atenção básica à saúde de 41 municípios com mais de 100 mil habitantes das regiões Sul e Nordeste do Brasil. A prevalência de participação foi de 62,7\%, sendo significativamente maior no Nordeste e no Programa Saúde da Família (PSF). A prática mais realizada foi educação em saúde em datas festivas, palestras e grupos. Verificou-se associação positiva do desfecho com sexo (feminino), capacitação no manejo de doenças crônicas, utilização de protocolos, satisfação com reuniões comunitárias e unidade de saúde com ensino. As diferenças da prevalência do desfecho por região e modelo reafirmam as expectativas inerentes ao contexto histórico da reorganização da atenção básica à saúde. Os resultados indicam que é necessário ampliar a participação dos trabalhadores de saúde junto à comunidade, considerando que a atenção básica à saúde é o nível de atenção que oferece a possibilidade de construção de práticas equânimes em saúde.

Prática Profissional; Pessoal de Saúde; Atenção Primária à Saúde

\section{Colaboradores}

A. R. Martins, D. S. Silveira e F. V. Siqueira participaram da concepção do artigo, análise de dados, interpretação e redação final do artigo. L. A. Facchini, R. X. Piccini, E. Tomasi, E. Thumé e M. C. Soares participaram na revisão crítica e redação final do manuscrito.

\section{Agradecimentos}

Os autores agradecem aos gestores, coordenadores de atenção básica à saúde e do Programa Saúde da Família, e aos trabalhadores das unidades básicas da saúde pelo apoio prestado para a realização do estudo. O presente artigo integra o Componente 3 do Projeto de Expansão e Consolidação do Saúde da Família, financiado pelo Ministério da Saúde e o Banco Mundial. 
Referências

1. Ministério da Saúde. Portaria no. 648, de 28 de março de 2006. Aprova a Política Nacional de Atenção Básica estabelecendo a revisão das diretrizes e normas para a organização da Atenção Básica para o Programa de Saúde da Família (PSF) e Programa de Agentes Comunitários de Saúde (PACS). Diário Oficial da União 2006; 29 mar.

2. World Health Organization. Primary health care. Geneva: World Health Organization; 1978.

3. Starfield B. Atenção primária: equilíbrio entre necessidades de saúde, serviços e tecnologia. Brasília: Organização das Nações Unidas para a Educação, a Ciência e a Cultura/Ministério da Saúde; 2002.

4. Paim JS. Modelos de atenção e vigilância da saúde. In: Rouquaryol MZ, Almeida FN, organizadores. In: Epidemiologia \& saúde. Rio de Janeiro: Editora Medsi; 2003. p. 567-86.

5. Ministério da Saúde. Saúde da família: uma estratégia para reorientação do modelo assistencial. Brasília: Ministério da Saúde; 1997.

6. Mattos RA. Os sentidos da integralidade: algumas reflexões acerca de valores que merecem ser defendidos. In: Pinheiro R, Mattos RA, organizadores. Os sentidos da integralidade na atenção e no cuidado à saúde. Rio de Janeiro: ABRASCO; 2001. p. 39-64.

7. Pedrosa JIS, Teles JBM. Consenso e diferenças em equipes do Programa Saúde da Família. Rev Saúde Pública 2001; 35:303-11.

8. Canesqui AM, Spinelli MAS. Saúde da família no Estado de Mato Grosso, Brasil: perfis e julgamentos dos médicos e enfermeiros. Cad Saúde Publica 2006; 22:1881-92.

9. Motta PR. Desempenho em equipes de saúde: manual. Rio de Janeiro: Editora FGV; 2001.

10. Gil CRR. Formação de recursos humanos em saúde da família: paradoxos e perspectivas. Cad Saúde Pública 2005; 21:490-8.

11. Carneiro Junior N, Andrade MC, Luppi CG, Silveira C. Organização de práticas de saúde equânimes em atenção primária em Região Metropolitana no contexto dos processos de inclusão e exclusão social. Saúde Soc 2006; 15:30-9.

12. Melo EM, Paiva L, Álvares J, Flecha ALD. A organização da Atenção Básica em municípios integrantes do Projeto de Expansão e Consolidação do Saúde da Família em Mato Grosso, Brasil. Cad Saúde Pública 2008; 24 Suppl l:S29-41.

13. Cotta RMM, Schott M, Azeredo CM, Franceschini SCC, Priore SE, Dias G. Organização do trabalho e perfil dos profissionais do Programa Saúde da Família: um desafio na reestruturação da atenção básica em saúde. Epidemiol Serv Saúde 2006; 15: 7-18.

14. Reis MAS, Fortuna CM, Oliveira CT, Durante MC. A organização do processo de trabalho em uma unidade de saúde da família: desafios para a mudança das práticas. Interface Comun Saúde Educ 2007; 11:655-66.

15. Shimizu HE, Dytz JLG, Lima MG, Moura AS. A prática do auxiliar de enfermagem do Programa Saúde da Família. Rev Latinoam Enferm 2004; 12: 713-20.
16. Ermel RC, Fracolli LA. O trabalho das enfermeiras no Programa de Saúde da Família em Marília/SP. Rev Esc Enferm USP 2006; 40:533-9.

17. Nascimento MS, Nascimento MAA. Prática da enfermeira no Programa de Saúde da Família: a interface da vigilância da saúde versus as ações programáticas em saúde. Ciênc Saúde Coletiva 2005; 10:333-45.

18. Ministério da Saúde. PROESF - Projeto de Expansão e Consolidação do Saúde da Família. Brasília: Ministério da Saúde; 2003.

19. Facchini LA, Piccini RX, Tomasi E, Thumé E, Teixeira VA, Silveira DS, et al. Avaliação de efetividade da Atenção Básica à Saúde em municípios das regiões Sul e Nordeste do Brasil: contribuições metodológicas. Cad Saúde Pública 2008; 24:159-72.

20. Levy P, Lemeshow S. Sampling for health professionals. Belmont: Lifetime Learning Publications; 1980.

21. Lemeshow S, Hosmer D. Adequacy of sample size in health studies. Chichester: John Wiley and Sons; 1990.

22. Lwanga S, Lemeshow S. Sample size determination in health studies: a practical manual. Geneva: World Health Organization; 1991.

23. Mari JJ, Iacoponi E, Williams P, Simões O, Silva JBT. Distúrbios psiquiátricos e assistência primária à saúde no Brasil. Rev Saúde Pública 1987; 21:501-7.

24. Starfield B. Global health, equity, and primary care J Am Board Fam Med 2007; 20:511-3.

25. Oliveira RG, Marcon SS. Trabalhar com famílias no Programa Saúde da Família: a prática do enfermeiro em Maringá - Paraná. Rev Esc Enferm USP 2007; 41:65-72.

26. Gil CRR. Atenção primária, atenção básica e saúde da família: sinergias e singularidades do contexto brasileiro. Cad Saúde Pública 2006; 22:1171-81.

27. Escorel S, Giovanella L, Mendonça MHM, Senna MCM. O Programa de Saúde da Família e a cons trução de um novo modelo para a atenção básica no Brasil. Rev Panam Salud Pública 2007; 21: 164-76.

28. Brasil. Lei no ${ }^{\circ} 8.080$ de 19 de setembro de 1990. Dispõe sobre as condições para a promoção, proteção e recuperação da saúde, a organização e funcionamento dos serviços correspondentes a dá outras providências. Diário Oficial da União 1990; 20 set.

29. Bodstein R. Atenção básica na agenda da saúde. Ciênc Saúde Coletiva 2002; 7:401-12.

30. Crevelin MA, Peduzzi M. A participação da comunidade na equipe de saúde da família. Como estabelecer um projeto comum entre trabalhadores e usuários? Ciênc Saúde Coletiva 2005; 10:323-31.

31. Ministério da Saúde. Avaliação da implementação do Programa Saúde da Família em 10 grandes centros urbanos: síntese dos principais resultados. Brasília: Ministério da Saúde; 2005.

32. Peterson JA. Get moving! Physical activity counseling in primary care. J Am Acad Nurse Pract 2007; 19:349-57.

33. Siqueira FV, Nahas MV, Facchini LA, Silveira DS, Piccini RX, Tomasi E, et al. Aconselhamento para a prática de atividade física como estratégia de educação à saúde. Cad Saúde Pública 2009; 25:203-13. 
34. Puig Ribeira A, McKenna J, Riddoch C. Attitudes and practices of physicians and nurses regarding physical activity promotion in the Catalan primary health-care system. Eur J Public Health 2005; 15:569-75.

35. Barbosa AAA, Brito EWG, Costa ICC. Saúde bucal no PSF, da inclusão ao momento atual: percepcões de cirurgiões dentistas e auxiliares no contexto de um município. Ciênc Odontol Bras 2007; 10:53-60.
36. Ministério da Saúde. Diretrizes da Política Nacional de Saúde Bucal. Brasília: Ministério da Saúde; 2004.

37. Machado MH. Perfil dos médicos e enfermeiros do Programa Saúde da Família no Brasil. Brasília: Ministério da Saúde; 2000.

Recebido em 15/Dez/2009

Versão final reapresentada em 20/Mar/2010

Aprovado em 19/Ago/2010 